\title{
A REMARK ON PICARD'S THEOREM
}

\author{
BY LENNART CARLESON
}

Communicated by P. R. Garabedian, October 3, 1960

1. In a recent manuscript $K$. Matsumoto has proved that there exists no general Picard theorem for functions meromorphic outside a set of logarithmic capacity zero. More precisely: given a closed set $E$ of logarithmic capacity zero, then there exists another closed set $F$ of capacity zero and a function $f(z)$, meromorphic in the complement of $F$ and with essential singularities at all points of $F$, such that $f(z)$ omits the set $E$. This result indicates that there is no Picard theorem at all for any perfect set. We shall here show that there still is an essential problem left, by giving an example of a set $F$ for which a strong Picard's theorem holds. ${ }^{1}$

TheOREM. There exists a linear closed set of positive capacity such that every function $f(z)$, meromorphic outside $F$ and omitting 4 values is rational.

2. We construct $F$ as a Cantor set on $(0,1)$ where the successive ratios $\xi$, decrease and satisfy the condition

$$
\lim _{\nu=\infty} \frac{\log \xi_{\nu}}{\log \nu}=-\infty
$$

Since $F$ is of positive capacity if and only if

$$
\sum_{1}^{\infty} \frac{\log \xi_{\nu}^{-1}}{2^{v}}<\infty
$$

$\xi_{\nu}$ can be chosen so that cap $(F)>0$. Let $f(z)$ be meromorphic outside $F$ and assume that $f(z) \neq a_{1}, a_{2}, a_{3}, a_{4}$. Define $f_{v}=\left(1-f a_{v}\right)\left(f-a_{v}\right)^{-1}$.

For the functions $f_{v}$, the following lemmas hold. $M$ denotes constants only depending on $a_{v}$.

LEMMA 1. If $f_{v}$ is holomorphic in $\rho \leqq|z-a| \leqq 2 \rho$ and $\left|f_{\nu}\left(z_{0}\right)\right| \leqq M$, $\left|z_{0}-a\right|=3 \rho / 2$, then $\left|f_{\nu}(z)\right| \leqq M$ for all $z, 9 \rho / 8 \leqq|z-a| \leqq 15 \rho / 8$.

Proof. This is Schottky's theorem.

LEMMA 2. If $f_{\nu}(z)$ is holomorphic in $\rho \leqq|z-a| \leqq K \rho, K \geqq 2$, and $\left|f_{\nu}(z)\right|$ is $<M$, then the circle $|z-a|=K^{1 / 2} \rho$ is mapped on a set of diameter $<M K^{-1 / 2}$.

1 This type of strong Picard theorem was used by $\mathrm{O}$. Lehto, $A$ generalization of Picard's theorem, Ark. Mat. vol. 3 (1958) p. 495. 
Proof. We consider $g(z)=f(\rho(z-a))$ in $1 \leqq|z| \leqq K$ and estimate $g^{\prime}(z)$ on $|z|=K^{1 / 2}$ by means of the Cauchy integral.

3. Let $I_{n-1}$ be an interval in the $(n-1)$ st subdivision of $(0,1)$. In the next construction, $I_{n-1}$ is divided into $I_{n}$ and $I_{n}^{\prime}$ of lengths $l_{n}$ and a complementary interval $\omega_{n}$. With the midpoint of $I_{n}$ as center, we construct the following circles:

$$
\begin{aligned}
& C_{n} \text { with radius } l_{n}, \\
& \Gamma_{n} \text { with radius } \frac{1}{3} l_{n-1}, \\
& \gamma_{n} \text { with radius }\left(\frac{1}{3} l_{n} l_{n-1}\right)^{1 / 2},
\end{aligned}
$$

$C_{n}^{\prime}, \Gamma_{n}^{\prime}, \gamma_{n}^{\prime}$ are the corresponding circles for $I_{n}^{\prime}$.

By Lemma 1 we have for, e.g., $\nu=1,2,3$,

$$
\left|f_{\nu}(z)\right|<M, \quad z \in \Gamma_{n}, \Gamma_{n}^{\prime} \quad(\nu=1,2,3)
$$

and for at least two indices

$$
\left|f_{\nu}(z)\right|<M, \quad z \in C_{n} \quad(\text { e.g., } \nu=1,2)
$$

and

$$
\left.\left|f_{\nu}(z)\right|<M, \quad z \in C_{n}^{\prime} \quad \text { (e.g., } \nu=1,3\right) .
$$

According to Lemma 2 there exist complex numbers $b$ and $b^{\prime},|b|$ $<M,\left|b^{\prime}\right|<M$, such that

$$
\left|f_{1}-b\right|<M /\left(K_{n}\right)^{1 / 2} \text { on } \gamma_{n}
$$

and

$$
\left|f_{1}-b^{\prime}\right|<M /\left(K_{n}\right)^{1 / 2} \text { on } \gamma_{n}^{\prime} \text {, }
$$

where

$$
K_{n}=\left(\frac{l_{n-1}}{l_{n}}\right)^{1 / 2}=\xi_{n}^{-1 / 2} .
$$

We now assume that

$$
\left|f_{1}-a\right|<\epsilon_{n-1} \text { on } C_{n-1}
$$

and consider the function

$$
g(z)=\left(f_{1}-a\right)\left(f_{1}-b\right)\left(f_{1}-b^{\prime}\right),
$$


in the region $R$ bounded by $C_{n-1}, \gamma_{n}$ and $\gamma_{n}^{\prime}$. On the boundary of $R$,

$$
|g(z)|<M \epsilon_{n-1}+M /\left(K_{n}\right)^{1 / 2}
$$

which, by the maximum principle implies

$$
\left|f_{1}-a\right|<M\left(\epsilon_{n-1}\right)^{1 / 3}+M /\left(K_{n}\right)^{1 / 6}
$$

in the region $R$. The same inequality holds for $b$ and $b^{\prime}$.

One function, e.g., $f_{2}(z)$, is bounded on $\gamma_{n}$ and the $C$-curves $C_{n+1}$ and $C_{n+1}^{\prime}$ inside $\gamma_{n}$. It follows from (3.2), (3.3) and (3.4) that there exists $c$ so that

$$
\left|f_{2}-c\right|<M /\left(K_{n}\right)^{1 / 2} \text { on } \gamma_{n} \text {. }
$$

If $\omega_{n}(z)$ is harmonic between $\gamma_{n} C_{n+1}^{\prime}$ and $C_{n+1}, \omega_{n}=1$ on $\gamma_{n}$ and $\omega_{n}=0$ on $C_{n+1}^{\prime}$ and $C_{n+1}$, an explicit calculation shows that $\omega_{n}>1 / M$ on $C_{n}$ since $\xi_{\nu}$ decreases. It follows from (3.5) that

$$
\log \left|f_{2}-c\right|<-\frac{\log K_{n}}{M} \text { on } C_{n} \text {. }
$$

Hence it follows that for certain $\alpha_{\nu}$ and a fixed $\eta>0$

$$
\left[f_{\nu}, \alpha_{\nu}\right] \leqq K_{n}^{-\eta} \text { on } C_{n},
$$

where [,] denotes cordal distance. Further $\epsilon_{n}<M K_{n}^{-\eta}$. Since $\sum K_{n}^{-\eta}$ $<\infty$ for all $\eta>0$, it follows that $f(z)$ is continuous as a function to the Riemann sphere. Hence $f(z)$ is rational, since $F$ is removable for bounded holomorphic functions.

UPPSAla UNIVERSITY, SWEDEN 